\title{
Case Report: Effects of Lower Thoracic Spinal Cord Stimulation on Bowel Management in
}

\section{a Person with Spinal Cord Injury}

\author{
Anthony F DiMarco ${ }^{1,2, *}$, Robert T Geertman ${ }^{3}$, Kutaiba Tabbaa ${ }^{4}$, Gregory A Nemunaitis ${ }^{1}$ and Krzysztof E Kowalski ${ }^{2,5,6}$ \\ ${ }^{1}$ Department of Physical Medicine and Rehabilitation, Case Western Reserve University, MetroHealth System, Ohio, USA \\ ${ }^{2}$ Department of Research, Case Western Reserve University, MetroHealth System, Ohio, USA \\ ${ }^{3}$ Department of Neurosurgery, Case Western Reserve University, MetroHealth System, Ohio, USA \\ ${ }^{4}$ Department of Anesthesiology, Case Western Reserve University, MetroHealth System, Ohio, USA \\ ${ }^{5}$ Department of Medicine, Case Western Reserve University, MetroHealth System, Ohio, USA \\ ${ }^{6}$ Research Service, Louis Stokes Cleveland VA Medical Center, Cleveland, Ohio, USA
}

* Corresponding author: Anthony F DiMarco, Department of Physical Medicine and Rehabilitation, Case Western Reserve University, MetroHealth Medical Center, Rammelkamp Center for Education and Research, Ohio, USA, Tel: 216-778-3906; Fax: 216-778-8589; E-mail: afd3@case.edu

Received: 22 Apr, 2019 | Accepted: 14 Jun, 2019 | Published: 21 Jun, 2019

Citation: DiMarco AF, Geertman RT, Tabbaa K, Nemunaitis GA, Kowalski KE (2019) Case Report: Effects of Lower Thoracic Spinal Cord Stimulation on Bowel Management in a Person with Spinal Cord Injury. J Neurol Neurobiol 5(1): dx.doi.org/10.16966/2379-7150.156

Copyright: (C) 2019 DiMarco AF, et al. This is an open-access article distributed under the terms of the Creative Commons Attribution License, which permits unrestricted use, distribution, and reproduction in any medium, provided the original author and source are credited.

\section{Abstract}

Background: Disturbances in bowel function occur in over $80 \%$ of persons with spinal cord injury $(\mathrm{SCl})$. Some of the challenges relate to their dependence on caregiver support, need for medications, and extensive time requirements of bowel management (BM). Lower thoracic spinal cord stimulation (SCS) has been shown to restore an effective cough. Participants also reported an improvement in bowel function, as well. However, this was not studied prospectively.

Objective: To determine whether usage of SCS to restore cough may improve BM in individuals with SCI.

Methods: In a participant with C6 AIS A tetraplegia, SCS was applied at home, 2-3times/day, on a chronic basis and as needed for secretion management and to facilitate BM. Stimulus parameters were set at values resulting in near maximum airway pressure generation $(P)(30-40 \mathrm{~V}, 50$ $\mathrm{Hz}, 0.2 \mathrm{~ms}$ ). P was measured at total lung capacity as an index of expiratory muscle strength. Questionnaires related to BM, were collected.

Results: $\mathrm{P}$ during spontaneous effort was $21 \mathrm{cmH}_{2} \mathrm{O}$. Following a period of reconditioning, SCS resulted in $\mathrm{P}$ of $144 \mathrm{cmH}_{2} \mathrm{O}$ and $172 \mathrm{cmH} \mathrm{O}_{2}$, at functional residual capacity (FRC) and total lung capacity (TLC) respectively. Over the course of $20 \pm 1$ week, BM time was reduced from 120 minutes (Pre-Implant) to 25 minutes. A mechanical method for BM (digital rectal stimulation and/or manual evacuation) was no longer needed. The number and dosages of medications required for BM was also reduced. This subject also reported marked subjective improvement in their BM routine. No complications of SCS were noted.

Conclusion: Our results suggest that SCS to restore cough may be a useful method to improve BM for both SCl individuals and their caregivers.

Keywords: Spinal Cord Injury; Tetraplegia; Bowel Management; Rehabilitation

Abbreviations: FRC: Functional Residual Capacity; TLC: Total Lung Capacity; SCS: Spinal Cord Stimulation; O: Oral Medications; R: Rectal Medications; b.i.d: Twice a Day; q.a.d: Every Other Day; q.i.d: 4 Times a Day; q.d: Daily

\section{Introduction}

Disturbances in bowel function occur in more than $80 \%$ of persons with spinal cord injury [1]. One of the major issues includes the inability to evacuate their bowels when defecation is convenient. More than half of individuals with an injury above L2 suffer from constipation; and, bowel routines, usually performed several times/ week, are inordinately time consuming for both individuals and their caregivers [2]. Moreover, these individuals often require both medications and mechanical methods to address bowel management (BM) concerns.
In an on-going clinical trial to restore an effective cough in tetraplegic individuals, spinal cord stimulation (SCS) is applied over the dorsal lower thoracic spinal cord. SCS results in marked contraction of the expiratory muscles which include the abdominal muscles, resulting in the generation of very large intra-abdominal pressures and restoration of an effective cough [3-9].

Over the course of this study, several persons volunteered that they became aware of an improvement in their bowel routines as a beneficial side effect consequent to the use of SCS. For this reason, we altered our clinical protocol to address BM before and after the 
application of SCS to restore cough.

This case report represents the first person in which BM following SCS was systematically addressed. These results demonstrate that SCS can have a significant beneficial clinical impact on BM including reductions in medications and mechanical methods and substantial reductions in the time required to complete their bowel routines.

\section{Methods}

This interventional clinical trial (Clinical Trials Registry: NCT01659541) was approved by the Investigational Review Boards at MetroHealth Medical Center and the National Institute of Neurological Disorders and Stroke. The participant provided written informed consent. His spontaneous vital capacity was 3.5 liters $(75 \%$ predicted). He had evidence of significant paresis of his expiratory muscles as evidenced by a maximum expiratory pressure generation (21 $\mathrm{cmH}_{2} \mathrm{O}, 9 \%$ predicted).

\section{Surgical procedure to place spinal cord wire electrodes to restore cough}

Two spinal cord wires leads, each with two electrode contacts, were inserted percutaneously through a needle and positioned in parallel. Each lead was advanced such that the upper electrode was positioned near the T9 spinal level on the dorsal epidural surface of the spinal cord. Given the distance between electrodes on each lead, the lower electrode was positioned near the T11 spinal level. Each lead was connected to a radiofrequency receiver (Finetech Medical Ltd, Welwyn Garden City, Herfordshire, United Kingdom), which was implanted anteriorly in a subcutaneous pocket over the upper chest wall $[3,5]$. There were no postoperative complications. Maximum expiratory pressure $(\mathrm{P})$ was measured using standard techniques with the airway occluded. Peak expiratory flow (F) was measured following release of occlusion.

Electrical stimulation was applied by activating a small portable external transmitter connected to an antenna, which was secured to the skin over the implanted receiver [5]. The system was designed to provide bipolar stimulation at the T9-T11 levels. The transmitter was activated by pushing a small button on the device. Since the expiratory muscles were significantly atrophied secondary to disuse, a period of repeated muscle stimulation was necessary to restore muscle strength. After an initial evaluation session, subjects applied SCS every 30 seconds for 5-10 minutes, 2 or 3 times/day, in the outpatient setting. Stimulus parameters were set at values resulting in maximal P generation. Subjects were also instructed to use the device for evacuation of secretions or airway clearance. This subject also used the device during his bowel routine.

A BIOPAC Data Acquisition and Analysis System with AcqKnowledge software, MP150 system with TSD 160C pressure transducer and TSD117 pneumotach airflow transducer interfaces with the DA 100C transducer amplifiers (Biopac Systems Inc, 42 Aero Camino, CA) was used to monitor online F and P. During the initial phase of stimulation, vital signs were closely monitored. Blood pressure was monitored carefully following the initial application of SCS. Based upon our protocol, if absolute systolic blood pressure exceeded $140 \mathrm{mmHg}$ systolic or $90 \mathrm{mmHg}$ diastolic, stimulation was stopped until values returned to baseline or less than 140 systolic or 90 $\mathrm{mmHg}$ diastolic. In this subject, blood pressure never exceeded 140 $\mathrm{mmHg}$ or $90 \mathrm{mmHg}$ diastolic.

This subject recorded the details of his BM for a 2-week period prior to implantation of the electrodes and for 28 weeks following initiation of SCS. This included duration of BM time, medications required and mechanical methods employed. A questionnaire rating the degree of change in his BM was also completed.

\section{Results}

The clinical data of the participant is provided in table 1 . Per protocol for entrance criteria of the study to restore of cough, the person had evidence of marked expiratory muscle weakness and associated reduction in peak expiratory flow.

Following daily SCS, P generation gradually increased over the first 8-14 weeks due to muscle reconditioning and then plateaued (Figure 1). With maximum stimulation $(40 \mathrm{~V}, 50 \mathrm{~Hz}, 200 \mu$ s pulse width) and concomitant participant effort, $\mathrm{P}$ increased to over $200 \mathrm{cmH}_{2} \mathrm{O}$ at TLC which is comparable to that achievable by normal individuals. The relationship between stimulus voltage and $\mathrm{P}$ is shown in figure 2 . Near maximal $P$ was generated with $30 \mathrm{~V}$. Since the difference between 30 and $40 \mathrm{~V}$ was small, this subject used $30 \mathrm{~V}$ on a chronic basis for evacuation of secretions, as needed.

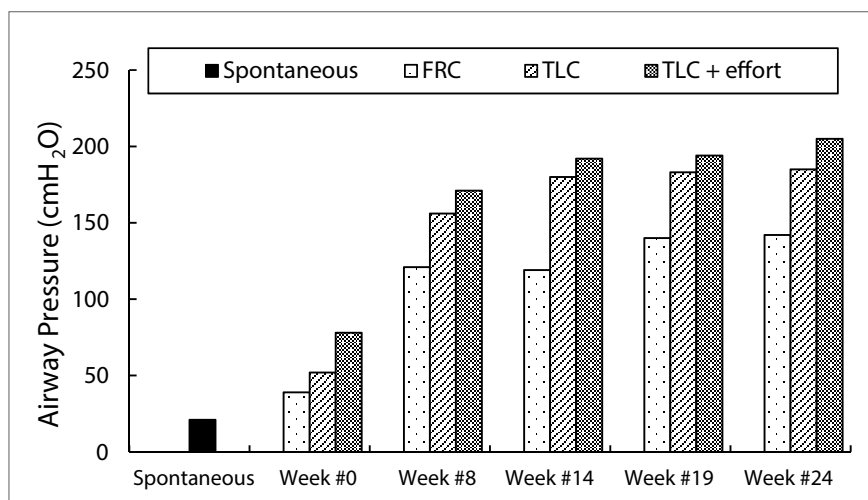

Figure 1: Maximum airway pressure generation (at FRC and TLC, with and without participant effort) during SCS (40 V, $50 \mathrm{~Hz}, 200 \mu \mathrm{s})$ at various times during the reconditioning period. Following daily SCS, airway pressure generation gradually increased over the first 8-14 weeks due to muscle reconditioning and then plateaued.

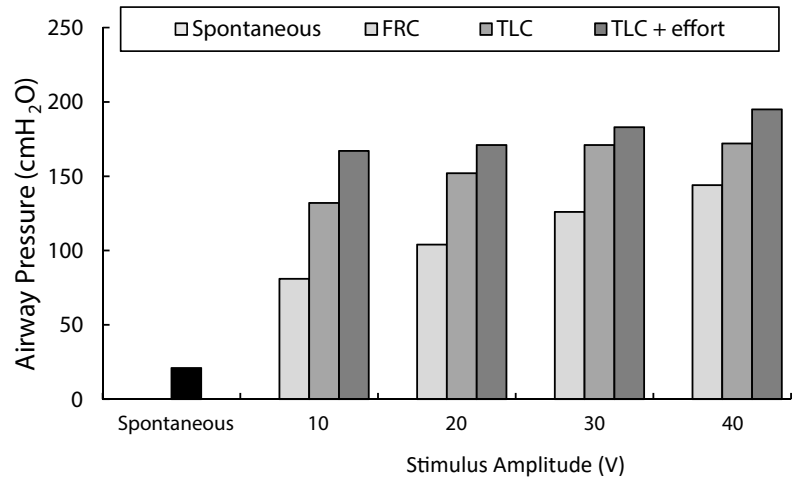

Figure 2: Relationship between stimulus amplitude and airway pressure generation during SCS (at FRC and TLC, with and without participant effort) after 24 weeks. Spontaneous airway pressure generation is shown for comparison (solid bar). There were progressive increases in airway pressure generation with increasing stimulus amplitude. 
This subject self-determined the optimal regimen for application of lower thoracic SCS during his daily bowel routine. As shown in figure $3 \mathrm{~A}$, he applied $20 \mathrm{~V}$ stimulation for 2-7 minutes several times approximately every 5 minutes. By the $8^{\text {th }}$ week of SCS, the amount of time required for his bowel routine management decreased from $\sim 2$ hours to $45 \mathrm{~min}$ and fell further to 25-30 $\mathrm{min}$ in subsequent weeks, representing more than a 75\% decrease in BM time (Figure 3B).

Prior to SCS, this subject employed both mechanical means (digital rectal stimulation and/or manual evacuation) and medications (colace $200 \mathrm{mg}$ bid, senna $50 \mathrm{mg}$ qid and enemas (as a part of his bowel routine management (Table 2). By week 14, he no longer required mechanical methods and these were discontinued completely. By week \#14, his colace requirements decreased to $200 \mathrm{mg} /$ day; he continued to use senna and enemas as he did prior to the implant. Importantly, there were no episodes of bowel leakage over the course of the study.
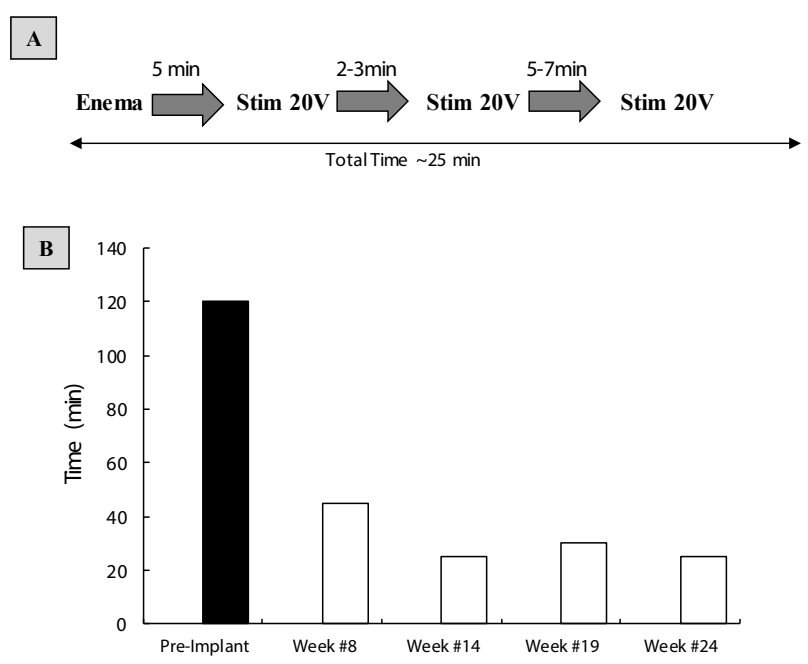

Figure 3A: Pattern of SCS used for bowel routines.

Figure 3B: Amount of time required for bowel management PreImplant (solid bar) and at various times during the reconditioning period (open bars). There was a significant reduction in the time required for bowel management after use of SCS.

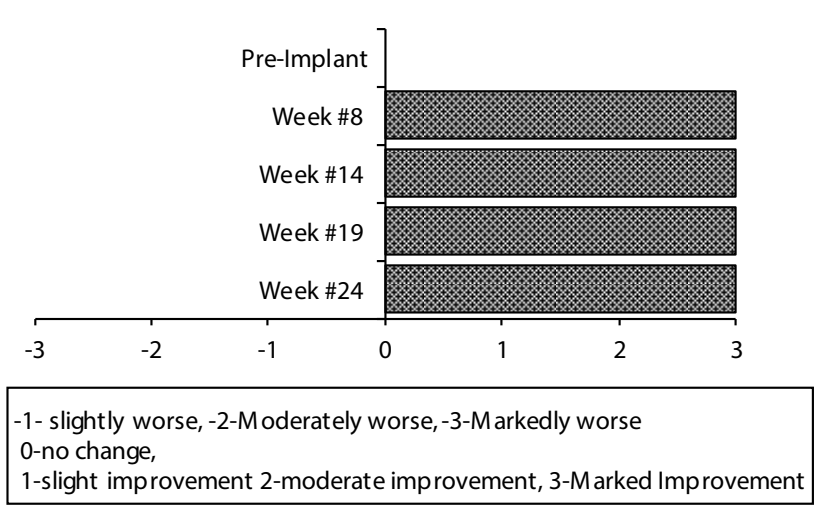

Figure 4: Subject assessment of bowel management. There was significant improvement at week \#8 and this persisted for the remainder of the 28-week study period.
Finally, as shown in figure 4, the subject rated the degree of change in his BM as marked improvement by week \#8 and this persisted for the remainder of the 28 -week study period.

\section{Discussion and Conclusion}

This case study is the first to demonstrate that use of SCS to restore cough has the beneficial side effect of improving BM in a person with complete cervical SCI. Importantly, the duration of the bowel routine was reduced substantially from 120 to 20-25 minutes. Since the bowel routine was applied 3-4 times/week, this represented a time saving of between 270-380 minutes/week ( 4.5 to $6.3 \mathrm{hrs} /$ week) for both the participant and caregiver. In addition, the needs for mechanical methods for bowel evacuation were no longer necessary and medication requirements were reduced. In addition, there was marked subjective improvement in his BM routine, as well.

The importance of this case report is highlighted by the fact that persons with SCI are more concerned with control of their intestinal function than with urinary control [10]. In fact, other investigators have suggested that decreasing time devoted to BM should be one of the key therapeutic goals in this person population [11].

Bowel programs are generally instituted in individuals with SCI and include oral laxatives, suppositories, enemas, digital stimulation, abdominal massage, Valsalva maneuver and manual evacuation [1]. While these methods have been shown to result in some improvement in gastrointestinal symptoms in individuals with SCI, significant symptoms persist. For example, Ozisler Z, et al. [1] found that constipation rates improved from 62 to $46 \%$ and difficult evacuation decreased from 38 to $24 \%$ of participants. Importantly, in those individuals who noted improvement based upon neurogenic bowel dysfunction scoring, significant symptoms often persisted. Indeed, the subject of this case report had been employing most of the above-mentioned bowel program methodology but had persistent and significant difficulties with constipation and intestinal evacuation.

Since many persons with cervical SCI have paralysis of their expiratory muscles, they are unable to generate large intra-abdominal pressures typically employed during normal defecation. These pressures generally range between 135 and $270 \mathrm{cmH}_{2} \mathrm{O}$ [12]. Expiratory muscle paralysis therefore likely represents an important factor in the frequent complaints of constipation in this person population. Consistent with this notion, Valles $\mathrm{M}$, et al. [11] evaluated participants with motor complete SCI and found that individuals with SCI with a neurological level above T7 without voluntary control of abdominal muscles is characterized by very frequent constipation $(86 \%)$ and infrequent incontinence. These symptoms resulted from a moderate delay in colonic transit time, incapacity to increase intra-abdominal pressure and absence of anal relaxation during the defecatory maneuver.

Other studies have also reported the more common occurrence of constipation in individuals with SCI. For example, Ozisler Z, et al. [1] reported constipation and incontinence rates of 56.6 and $41.8 \%$, respectively in motor complete SCI. Han TR, et al. [13] and other investigators [11,14-16] also reported constipation and difficult intestinal evacuation as the most common gastrointestinal problems. Interestingly, the subject of this case report did not report incontinence as a concern either before institution of SCS nor afterwards. Moreover, in our previous case series, none of our persons participating in the study of SCS to restore cough reported fecal incontinence associated with SCS [3-9].

Similar to prior studies of SCS to restore cough, this method resulted in the generation of large positive airway pressures which in 
Table 1: Clinical data of the participant.

\begin{tabular}{|c|c|c|c|c|c|c|c|c|c|}
\hline Sex & $\begin{array}{l}\text { Age } \\
\text { (y) }\end{array}$ & $\begin{array}{l}\text { Weight } \\
\text { (kg) }\end{array}$ & $\begin{array}{l}\text { Cause of } \\
\text { Injury }\end{array}$ & $\begin{array}{l}\text { Level of } \\
\text { Injury }\end{array}$ & AIS & $\begin{array}{l}\text { Elapsed Time } \\
\text { Since Injury (y) }\end{array}$ & $\begin{array}{c}\text { Spontaneous Vital } \\
\text { Capacity (L) } \\
\text { (\% predicted) }\end{array}$ & $\begin{array}{l}\text { Peak Expiratory Airflow } \\
\text { (L/s) } \\
\text { (\% predicted) }\end{array}$ & $\begin{array}{c}\text { Maximal Expiratory } \\
\text { Pressure }\left(\mathrm{cm} \mathrm{H}_{2} \mathrm{O}\right) \\
\text { (\% predicted) }\end{array}$ \\
\hline$M$ & 35 & 93 & Fall & C6 & $A$ & 4.3 & $3.5(73 \%)$ & 2.7 (29\%) & 21 (9\%) \\
\hline
\end{tabular}

Table 2: Bowel routine management.

\begin{tabular}{|c|c|c|c|c|c|}
\hline Pre-Implant & Week \#8 & Weeke \#14 & Week \#19 & Week \#24 & Week \#30 \\
\hline \multicolumn{6}{|c|}{ Medications (laxatives) Used to Facilitate Bowel Routine } \\
\hline $\begin{array}{c}\text { O: Colace }(200 \mathrm{mg} \text {, } \\
\text { b.i.d.) }\end{array}$ & O: Colace (200 mg, b.i.d.) & $\begin{array}{l}\text { O: Colace }(200 \mathrm{mg} \text {, } \\
\text { b.i.d.) }\end{array}$ & $\begin{array}{l}\text { O: Colace }(200 \mathrm{mg} \text {, } \\
\text { q.d.) }\end{array}$ & $\begin{array}{c}\text { O: Colace }(200 \mathrm{mg} \text {, } \\
\text { q.d.) }\end{array}$ & $\begin{array}{c}\text { O: Colace }(200 \mathrm{mg} \text {, } \\
\text { q.d.) }\end{array}$ \\
\hline $\begin{array}{l}\text { O: Senna (50 mg (2 tab } \\
25 \text { mg each) q.i.d.) }\end{array}$ & $\begin{array}{l}\text { O: Senna (50 mg (2 tab } \\
25 \text { mg each) q.i.d.) }\end{array}$ & $\begin{array}{l}\text { O: Senna (50 mg (2 tab } \\
25 \text { mg each) q.i.d.) }\end{array}$ & $\begin{array}{l}\text { O: Senna (50 mg ( } 2 \\
\text { tab } 25 \text { mg each) q.d.) }\end{array}$ & $\begin{array}{l}\text { O: Senna ( } 50 \text { mg ( } 2 \\
\text { tab } 25 \text { mg each) q.d.) }\end{array}$ & $\begin{array}{l}\text { O: Senna (50 mg ( } 2 \\
\text { tab } 25 \text { mg each) q.d.) }\end{array}$ \\
\hline $\begin{array}{l}\text { R: Enema (<150 mL, } \\
\text { q.a.d) }\end{array}$ & $\begin{array}{l}\text { R: Enema (<150 mL, } \\
\text { q.a.d) }\end{array}$ & $\begin{array}{l}\text { R: Enema (<150 mL, } \\
\text { q.a.d) }\end{array}$ & $\begin{array}{l}\text { R: Enema (<150 mL, } \\
\text { q.a.d) }\end{array}$ & $\begin{array}{l}\text { R: Enema (<150 mL, } \\
\text { q.a.d) }\end{array}$ & $\begin{array}{l}\text { R: Enema (<150 mL, } \\
\text { q.a.d) }\end{array}$ \\
\hline $\begin{array}{l}\text { Mechanical methods } \\
\text { (digital rectal } \\
\text { stimulation and/or } \\
\text { manual evacuation) }\end{array}$ & $\begin{array}{l}\text { Mechanical methods } \\
\text { (digital rectal stimulation } \\
\text { and/or manual } \\
\text { evacuation) }\end{array}$ & No Mechanical & No Mechanical & No Mechanical & No Mechanical \\
\hline \multicolumn{6}{|c|}{ Average Frequency of Bovel Activities (per week) } \\
\hline 4/week & 4-5/week & 3/week & 3/week & 3/week & 3/week \\
\hline \multicolumn{6}{|c|}{ Amount of Time Required for Bowel Management (min) } \\
\hline 120 & 45 & 25 & 30 & 25 & 25 \\
\hline \multicolumn{6}{|c|}{ Amount of Time Required for Bowel Management (\%) } \\
\hline $100 \%$ & $37.50 \%$ & $20.80 \%$ & $25 \%$ & $20.80 \%$ & $20.80 \%$ \\
\hline
\end{tabular}

this subject approached $200 \mathrm{cmH}_{2} \mathrm{O}$ when performed at TLC [3-9]. Restoration of the ability to generate large intra-abdominal pressures by use of SCS therefore, likely played an important role in the improvements in bowel function observed by this subject.

As this is a single case report, the results are not generalizable to the SCI population at large. Going forward therefore, our plan is to evaluate additional individuals undergoing restoration of cough with SCS in terms of their bowel routine. Ultimately, a definitive study of the impact of SCS on potential improvements on bowel routines in this person population may be warranted.

\section{Acknowledgement}

This work was supported by the NIH-NINDS (U01 NS083696), CTSA (UL1TR000439). This investigation was approved by the Institutional Review Board of MetroHealth Medical Center (IRB1500014). Clinical Trials Registry: NCT01659541; FDA IDE: G980267.

\section{Conflict of Interest Notification}

Dr. DiMarco holds two United States Patents for technology related to the content of this paper: Method and Apparatus for Electrical Activation of the Expiratory Muscles to Restore Cough $(5,999,855)$; Bipolar Spinal Cord Stimulation to Activate the Expiratory Muscles to Restore Cough $(8,751,004)$.

\section{References}

1. Ozisler Z, Koklu K, Ozel S, Unsal-Delialioglu S (2015) Outcomes of bowel program in spinal cord injury patients with neurogenic bowel dysfunction. Neural Regen Res 10: 1153-1158.

2. De Looze D, Van Laere M, De Muynck M, Beke R, Elewaut A (1998) Constipation and other chronic gastrointestinal problems in spinal cord injury patients. Spinal Cord 36: 63-66.

3. DiMarco AF, Geertman RT, Tabbaa K, Kowalski KE (2019) Complete Restoration of Respiratory Muscle Function in Three Subjects with Spinal Cord Injury: Pilot Interventional Clinical Trial. Am J Phys Med Rehabil 98: 43-50.

4. DiMarco AF, Geertman RT, Tabbaa K, Polito RR, Kowalski KE (2017) Economic consequences of an implanted neuroprosthesis in subjects with spinal cord injury for restoration of an effective cough. Top Spinal Cord Inj Rehabil 23: 271-278.

5. DiMarco AF, Geertman RT, Tabbaa K, Polito RR, Kowalski KE (2018) Minimally Invasive Method to Activate the Expiratory Muscles to Restore Cough. J Spinal Cord Med 41: 562-566.

6. DiMarco AF, Kowalski KE, Geertman RT, Hromyak DR (2009) Lower thoracic spinal cord stimulation to restore cough in patients with spinal cord injury: results of a National Institutes of Healthsponsored clinical trial. Part I: methodology and effectiveness of expiratory muscle activation. Arch Phys Med Rehabil 90: 717. 
7. DiMarco AF, Kowalski KE, Geertman RT, Hromyak DR, Frost FS, et al. (2009) Lower thoracic spinal cord stimulation to restore cough in patients with spinal cord injury: results of a National Institutes of Health-sponsored clinical trial. Part II: clinical outcomes. Arch Phys Med Rehabil 90: 726-732.

8. DiMarco AF, Kowalski KE, Geertman RT, Hromyak DR (2006) Spinal cord stimulation: a new method to produce an effective cough in patients with spinal cord injury. Am J Respir Crit Care Med 173 1386-1389.

9. DiMarco AF, Kowalski KE, Hromyak DR, Geertman RT (2014) Longterm follow-up of spinal cord stimulation to restore cough in subjects with spinal cord injury. J Spinal Cord Med 37: 380-388.

10. Glickman S, Kamm MA (1996) Bowel dysfunction in spinal-cordinjury patients. Lancet 347: 1651-1653.

11. Vallès $M$, Vidal J, Clavé $P$, Mearin $F$ (2006) Bowel dysfunction in patients with motor complete spinal cord injury: clinical, neurological, and pathophysiological associations. Am J Gastroenterol 101: 2290 2299.

12. Carry PY, Banssillon V (1994) Intra-abdominal pressure. Ann Fr Anesth Reanim 13: 381-399.

13. Han TR, Kim JH, Kwon BS (1998) Chronic gastrointestinal problems and bowel dysfunction in patients with spinal cord injury. Spinal Cord 36: 485-490.

14. Demirel G, Soy D, Öztürk Y, Başoğlu I, Yılmaz H (1999) Spinal kord yaralanmalı hastalarda gastrointestinal sistem problemleri ve barsak fonksiyon bozuklukları. Romatol Tıp Rehab 10:186-189.

15. Stone JM, Nino-Murcia M, Wolfe VA, Perkash I (1990) Chronic gastrointestinal problems in spinal cord injury patients: a prospective analysis. Am J Gastroenterol 85: 1114-1119.

16. Krassioukov A, Eng JJ, Claxton G, Sakakibara BM, Shum S (2010) Neurogenic bowel management after spinal cord injury: a systematic review of the evidence. Spinal Cord 48: 718-733. 